\title{
Modeling and Measurement of Radiated Field Emission From a Power/Ground Plane Cavity Edge Excited by a Through-Hole Signal Via Based on a Balanced TLM and Via Coupling Model
}

\author{
Jun So Pak, Member, IEEE, Hyungsoo Kim, Student Member, IEEE, Junwoo Lee, Member, IEEE, and \\ Joungho Kim, Member, IEEE
}

\begin{abstract}
A balanced transmission line model (TLM) and via coupling model is proposed for efficient simulation of radiated field emission from a power/ground plane cavity edge, where the radiated field emission is excited by a through-hole signal via in a multilayer package and printed circuit board (PCB). The radiated field emission is simulated and measured with a series of test boards. The simulation agrees fairly well with the measurement confirming the preciseness and usefulness of the proposed model. It is shown that the through-hole signal via is a considerable source of the radiated field emission as well as the signal loss. When the signal trace is switching vertically stacked reference planes, the signal return current path is disconnected at the via and the impedance becomes extremely high. A significant amount of insertion loss and radiated field emission is generated at resonance frequencies of the plane cavity. The effect of a decoupling capacitor fence (De-Cap Fence) at the edge of the board to mitigate the radiated field emission is examined. The proposed model confirms that the De-Cap Fence changes the resonance mode and frequency of the plane cavity, and reduces the radiated field emission.
\end{abstract}

Index Terms-Decoupling capacitor, electromagnetic interference (EMI), impedance, power/ground plane, radiated field emission, simultaneous switching noise (SSN), stitching via, through-hole signal via, transmission line modeling (TLM), via.

\section{INTRODUCTION}

I $\mathrm{N}$ MODERN high-performance digital devices, packages, and systems, clock frequency and its harmonics are elevated to gigahertz frequency ranges. Furthermore, power supply and ground return current exceeds tens of amperes to drive numbers of I/O drivers and digital core circuits. The device, package, and system density is also high. The combined effect of the high-frequency current spectrum components and the high power supply

Manuscript received January 14, 2004; revised July 10, 2005 and May 14, 2006. This work was supported by the Center for Electronic Packaging Materials, Korea Science and Engineering Foundation.

J. S. Pak is with the High Density Interconnection Group, Nanoelectronics Research Institute, National Institute of Advanced Industrial Science and Technology (AIST), Ibaraki 305-8568, Japan (e-mail: chitoong@eeinfo.kaist.ac.kr; junso-pak@aist.go.jp).

H. Kim and J. Lee are with the Advanced Design Team, Research and Development Division, Hynix Semiconductor, Inc., Gyunggi-do 467-701, Korea (e-mail: hyungsoo2.kim@ @ynix.com; junwoo.lee@hynix.com).

J. Kim is with the Terahertz Interconnection and Package Laboratory, Division of Electrical Engineering, Department of Electrical Engineering and Computer Science, Korea Advanced Institute of Science and Technology (KAIST), Daejon 305-701, Korea (e-mail: joungho@ee.kaist.ac.kr).

Digital Object Identifier 10.1109/TADVP.2006.890210 and $\mathrm{I} / \mathrm{O}$ current raises serious design concerns and challenges in controlling the resulting radiated field emission [1], [2]. As the radiated field emission problems increase, they turn out to be more difficult to solve. In particular, the radiated field emission issues arising from multilayer interconnection structures for the high-density packages and printed circuit boards (PCBs) become troublesome because of the high integration density and the relatively large effective antenna size. There are several major metallic structures in the multilayer interconnections that can emit electromagnetic fields, including unshielded waveguides or transmission lines [3], attached cables [4], [5], metallic components such as a heat sink [6], and plane cavity edges [7]. Through the unceasing efforts of researchers, there is good understanding of the first three radiated field emission problems and their solution, including embedded strip line structures for signal and clock lines, electromagnetic interference (EMI) filtering methods, and metal encapsulation of the components. Recently, studies have been initiated to mitigate the radiated field emission from plane cavity edges [7], [10]-[16].

The multilayer power/ground plane stack-up is commonly used in high-speed packages and systems in order to accommodate feasible routings of the high-density interconnections and to lower power/ground network impedance. These structures naturally become electromagnetic resonance cavities; the resonance frequency is determined by the dielectric constant of the insulator and the physical size of the plane cavity [8]. When there is a source to excite the cavity resonance, the cavity becomes a significant source of radiated field emission from the power/ground plane edge as a form of an aperture antenna [7], [9]. One of the important sources of cavity resonance excitation is disrupted return current (discontinuity) at high-speed signal lines or clock lines as illustrated in Fig. 1 [11]-[14]. The return current is disrupted when the signal line and the clock line change their reference planes (power/ground planes) through the via. Then, the disrupted return current produces a high degree of voltage fluctuation at the reference planes around the via, where the line impedance becomes very high owing to the broken return current path. In particular, at cavity resonance frequencies, the line impedance becomes extremely high, causing a very large amount of voltage fluctuation between the reference planes and a strong high-frequency electric field at the edge of the power/ground plane cavity as a consequence. Another important source of cavity excitation is the simultaneous switching 

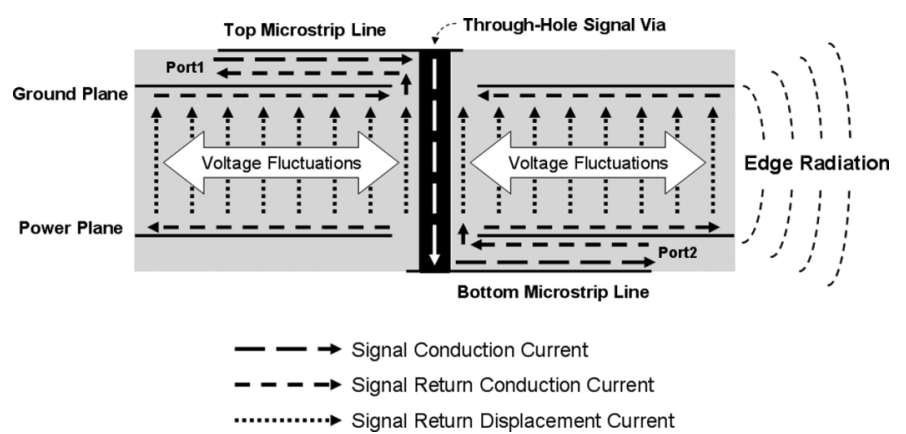

Fig. 1. Current distributions and voltage fluctuations induced by the broken return current path (discontinuity) of the through-hole signal via in a multilayer PCB. Currents are classified into conduction current (long-dashed line, and dashed line) and displacement current (dotted line). The displacement current mainly contributes voltage fluctuation in the power/ground plane and consequently produces the radiated field emission from the power/ground plane cavity edge.

current flowing through the power via or the ground via. In this case, these vias are electrically connected to a plane of the cavity [7], [10], [15], [16].

Simulation methods have been suggested to comprehend and to predict the radiated field emission from the power/ground plane edge in [10], [15], and [16]. An analytical model based on a Green's function of the rectangle-shaped power/ground plane is used to understand the fundamental relation between the power/ground network impedance and the radiated field emission from the power/ground plane cavity in [10] and [15]. This simulation is not effective if the shape of the power/ground plane cavity is changed to an arbitrary form, and it may not be applied to a complex power/ground plane structure with a large number of decoupling capacitors and vias. Another modeling method to simulate the radiated field emission from the power/ground plane cavity edge is the finite-difference time-domain (FDTD) method [16]. Even though the FDTD is a valuable modeling method for analyzing complex structures, it requires large simulation times and memory resources. Moreover, since the multilayer power/ground network has the smallest structure-like via and the largest structure-like plane, it is very difficult for the FDTD method to incorporate recent developments in complex high-density interconnection systems.

Another useful alternative to simulate the field emission phenomena from the power/ground cavity edge is transmission line modeling (TLM) [15]. Since TLM is based on a spice type circuit model, the modeling method can be easily embedded into the conventional SPICE-type circuit simulator, and the simulation time can be considerably reduced. Moreover, various passive and active device models can be readily inserted into the circuits of the TLM by including commonly used component models. Furthermore, it can be applied to arbitrary shapes of the power/ground planes using the unit cell concept. In this paper, we extend the conventional TLM method to describe the power/ground cavity resonance of the multiple cavity and transmission line structures, when the resonance is excited by a through-hole signal via. In our modeling approach, the TLM circuit has the form of a balanced circuit formation to explain the excitation mechanism of the cavity resonance by the disrupted return current when the signal line changes the refer- ence planes. The radiated field emission excited by the simultaneous switching power/ground current can be analyzed as a single plane problem with an ideal ground. On the other hand, in our study, the through-hole signal via is coupled equally to both the power plane and the ground plane of a cavity in a balanced manner, and we cannot assign a plane as a ground point. Furthermore, a via coupling model of the through-hole signal via is suggested to describe the electromagnetic coupling to the plane cavity. From the simulated electric field at the plane cavity edge, we calculate the radiated field emission using a magnetic current dipole at the cavity edge and antenna equations.

To verify the proposed model and simulation method, we have fabricated a series of test multilayer boards with variations in the through-hole signal via positions. We have measured the $S$-parameters and the radiated field emissions and compared them with the simulated expectations. In the first step, we have measured the $S$-parameters of the signal trace, which changes the reference planes through the through-hole signal via, and have successfully verified the proposed model by comparison with the $S$-parameter measurements. As expected, the signal suffers a very large amount of signal insertion loss at the power/ground plane cavity resonance frequencies. The amount of signal insertion loss and the positions of the resonance frequencies are closely predicted by the proposed model and the simulation method. The resonance frequencies are determined by the power/ground plane dimensions and the dielectric constant of the insulator, while the occurrence of the specific resonance is governed by the position of the through-hole signal via at the surface of the planes.

In the second step, we have measured the radiated field emissions from the edge of the test PCB and have compared them with the simulation. The measured radiated field emission spectrum from the power/ground plane cavity edge is well matched to the modeling and simulation results, which confirms the preciseness and usefulness of the model. We demonstrated that the through-hole signal via can be a considerable source of the edgeradiated field emission when the return current is disrupted. Through the modeling procedure, we were able to explain the excitation mechanism of the edge field emission from the power/ ground plane cavity excited by the through-hole signal via.

In the final step, we have investigated the ability of the decoupling capacitor fence (De-Cap Fence) at the cavity edge to mitigate the radiated field emission. As predicted by the proposed model, the De-Cap Fence changes the mode and the frequency of the plane cavity resonance, and reduces the radiated field emission by $7 \mathrm{~dB}$ at the most critical frequency. The De-Cap Fence has a similar structure to via stitching, which has been introduced in [15] and [16]. As the number of decoupling capacitors per unit length of the fence is increased, the amount of the edge radiation field emission is diminished.

\section{Proposed Balanced TLM AND Via Coupling Model}

The TLM is a field solution method for various waveguides by using well-known relationships between electromagnetic field quantities, and voltage and current in transmission lines [18]. Field patterns in a small area of the waveguides are the same as those of three short shunt transmission lines, which are located in Cartesian coordinates. If the waveguide can be spatially di- 


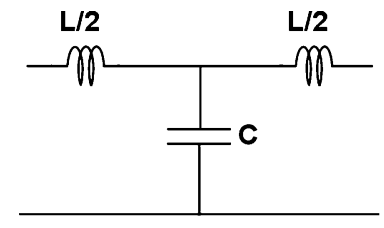

(a)

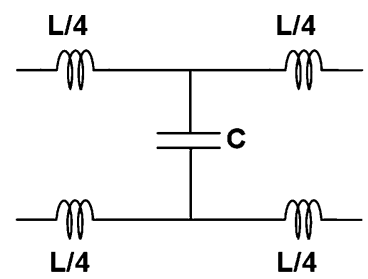

(b)
Fig. 2. Transmission line model used in (a) the conventional TLM and (b) the balanced TLM. Model parameters $(L$ and $C$ ) are determined in a TLM mesh, size $1 / 20$ wavelength of the maximum target frequency.

vided into the numbers of the small areas and each small area can be replaced by three transmission lines or their equivalent circuit models, TLM of the waveguide is obtained. In this respect, the small area represented by three transmission lines is called as a mesh or rectangular unit cell of TLM. If a transverse magnetic field (TM) to the $z$-axis is considered in planar structures located on the $x-y$ plane, such as multilayer interconnections in packages and PCBs, the TLM becomes an efficient two-dimensional analysis method and is the conventional TLM, as used in [15]. One of the advantages of the TLM is that even the most complicated structures can be analyzed because of its flexibility, which accomplishes model networking with the electromagnetic fields defined by the boundaries and materials. Another advantage is that any excitations, time domains or frequency domains at any position can be embedded into the TLM, which is constructed with spice circuit components.

The conventional TLM uses the transmission line model as shown in Fig. 2(a). Inductance $(L)$ and capacitance $(C)$ per TLM mesh size are included. In this paper, we used the balanced TLM to analyze the electromagnetic field distribution inside the power/ground plane cavity of the multilayer board when the field is excited by the through-hole signal via. The schematic of the proposed balanced TLM mesh is illustrated in Fig. 2(b). In the balanced TLM, total line inductance in a mesh is shared by the two inductors in the power and ground lines. The ground line inductance represents the inductor model at the return current path.

Through the conventional TLM analysis, the power/ground plane cavity impedance and the associated simultaneous switching noise (SSN) problems were studied [15]. However, the conventional TLM cannot be applied to problems of the power/ground plane cavity where the ground current path is no longer negligible. Such cases occur when there are slots or cutouts in the ground plane, producing high-impedance sections at the return current path of the signal line. In particular, when the signal trace is switching the reference planes through the signal via in the multilayer stack, the signal return current is disrupted at the via, and the impedance of the signal return current path becomes extremely high at the resonance frequencies of the power/ground plane cavity. Furthermore, in the multilayer power/ground stack, it is not practicable to assign a single node at a single ground plane as in the conventional TLM, since there are many ground planes connected by the vias and the decoupling capacitors.

Fig. 3 shows the circuit schematic of the proposed balanced TLM and via coupling model employed for the analysis of a

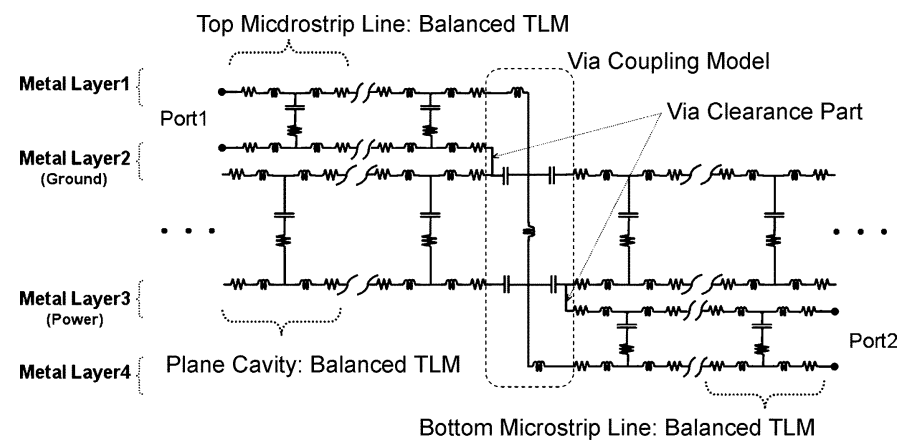

Fig. 3. Circuit schematic of the proposed balanced TLM and via coupling model employed for analysis of the four-layer PCB including a through-hole signal via.
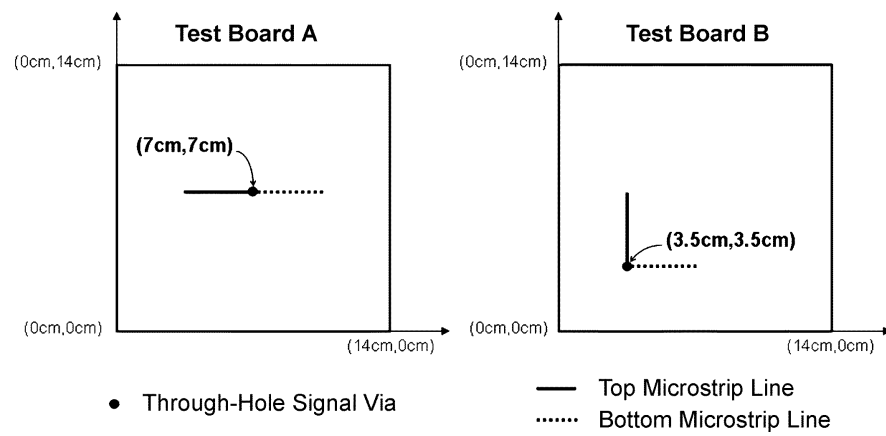

Fig. 4. Structure and dimensions of two test boards. Both are designed on the $14 \mathrm{~cm}$ by $14 \mathrm{~cm}$ power/ground plane pair. Test board A has one through-hole signal via at the center location $(7 \mathrm{~cm}, 7 \mathrm{~cm})$, and test board $\mathrm{B}$ has one at the offset location $(3.5 \mathrm{~cm}, 3.5 \mathrm{~cm})$. Two microstrip lines are connected by the through-hole signal via.

four-layer board including a through-hole signal via. The metal layer 2 is used as a reference ground plane, and it serves not only as a return current path for a microstrip signal line in metal layer 1, but also as a balanced current path for a cavity, which is formed by metal layer 2 and metal layer 3 . The two current paths at each surface of metal layer 2 are separated from each other by the skin depth of the high-frequency return currents. Consequently, both the microstrip signal line and the plane cavity are described by the two separated balanced TLM schematics, and their electrical connections are formed through only the via clearances (via clearance part) as shown in Fig. 3. The microstrip line model is a one-dimensional balanced TLM, and the plane cavity model is a two-dimensional balanced TLM. The proposed balanced TLM model can be easily extended to multilayer stack structures of more than four layers. In order to describe the return current path break at the signal via where the signal trace exchanges the reference planes, and in order to model the excitation of the power/ground plane cavity resonance, the via model is included as a form of coupling model to the plane cavity. The capacitive coupling is expressed as coupling capacitors connected between the via metal column and the via clearance part. The via column is described as an inductor, and the via neck effect is modeled as a series inductor to the signal line.

To verify the proposed balanced TLM and via coupling model, test boards were fabricated. The test boards were designed on a four-layer board using FR-4 insulator $\left(\varepsilon_{r}=4.35\right)$ 


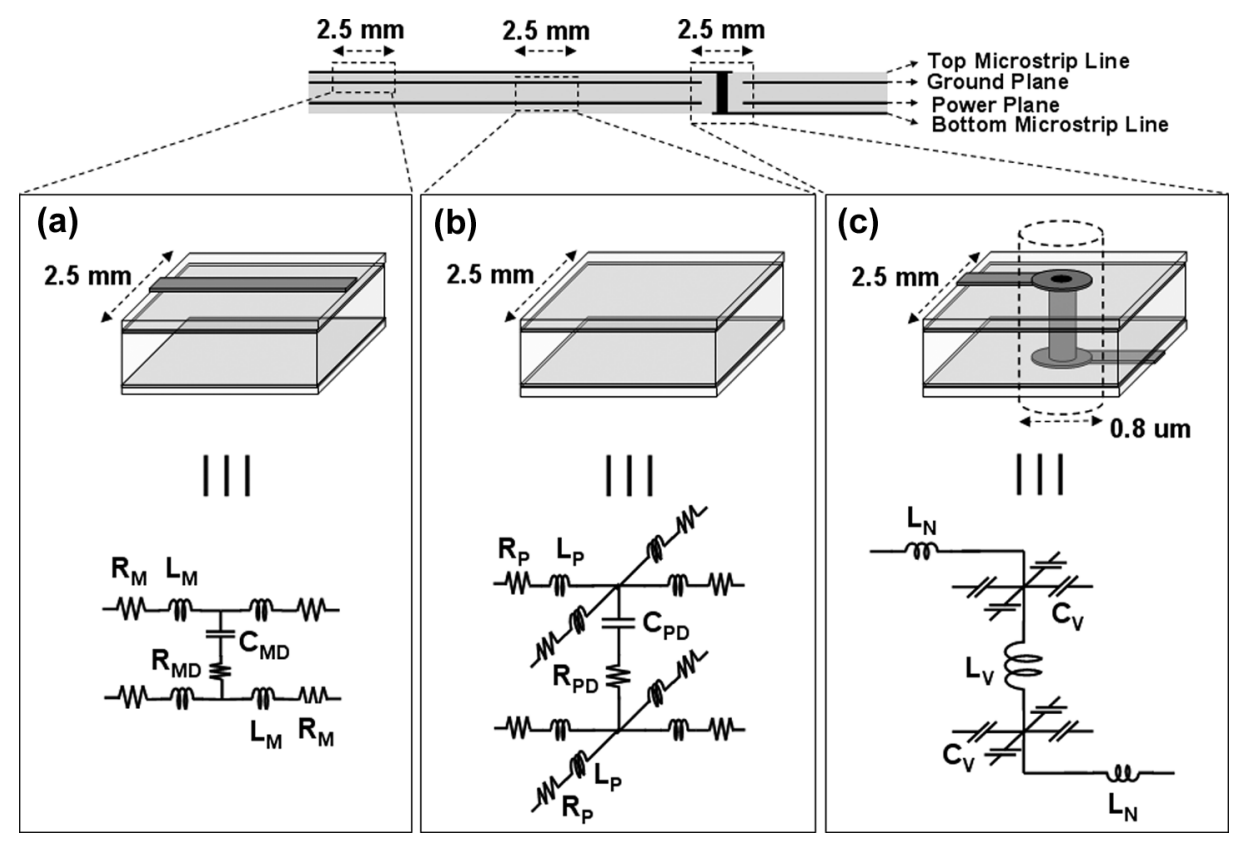

Fig. 5. TLM unit cells on the test board and their equivalent circuit models. The unit cells are classified into three. (a) shows the unit cell and the balanced TLM of microstrip line, (b) shows the unit cell and the balanced TLM of power/ground plane cavity, and (c) shows the unit cell of power/ground plane cavity including through-hole signal via and the via coupling model of through-hole signal via part (dashed line cylinder).

and 1-oz copper (thickness $=0.035 \mathrm{~mm}$ ). Top and bottom insulators are $0.1 \mathrm{~mm}$ thick, and the middle one is $1 \mathrm{~mm}$ thick. A through-hole signal via has a $0.3-\mathrm{mm}$ diameter drill hole, $0.6-\mathrm{mm}$ diameter via land pad, and $0.8-\mathrm{mm}$ diameter via clearance. These dimensions are typical in commonly used six-layer PCBs in commercial production. The inner two layers for strip lines were not used in the test boards. We have designed and tested two different test boards, which have two different via locations on the board. As seen in Fig. 4, test board A has a via location at $(7 \mathrm{~cm}, 7 \mathrm{~cm})$ and test board $B$ has a via location at $(3.5 \mathrm{~cm}, 3.5 \mathrm{~cm})$. The power/ground plane has a square shape $14 \mathrm{~cm}$ by $14 \mathrm{~cm}$. There are no other electrical connections, such as a stitching via or decoupling capacitor, between the planes in a cavity. The microstrip lines on metal layer 1 and metal layer 4 are $3.5 \mathrm{~cm}$ in length and $0.2 \mathrm{~mm}$ in width to maintain about $50-\Omega$ line impedance.

In this structure, one power/ground plane with 1-mm thickness was considered, even though nowadays multilayer PCB has more than two numbers of power/ground planes with thinner thickness, because there should be at least one power/ground pair whose thickness is compatible to $1 \mathrm{~mm}$.

Unit cells and their equivalent circuit models for the balanced TLM and via coupling model are represented in Fig. 5. To verify the model up to $3 \mathrm{GHz}$, the unit cell size was determined to be $2.5 \mathrm{~mm}$ by $2.5 \mathrm{~mm}$, which is close to $1 / 20$ of the $3-\mathrm{GHz}$ wavelength in FR-4. The balanced TLM of microstrip line and its parameters are seen in Fig. 5(a) and Table I. The microstrip line unit cell is composed of ten circuit elements, including four inductors $\left(L_{M}\right)$ and four resistors $\left(R_{M}\right)$ for the conduction current paths at the metal layer 1 (signal) and at the upper surface (reference) of metal layer 2 . The wide reference plane $(2.5 \mathrm{~mm})$ enables a balanced transmission line model, based on image
TABLE I

PARAMETERS OF THE BALANCED TLM OF MICROSTRIP LINE

\begin{tabular}{|c|c||c|}
\hline Notation & Value & Total Value \\
\hline $\mathbf{R}_{\mathbf{M}}$ & $6 \mathrm{~m} \Omega$ & $24 \mathrm{~m} \Omega /$ Cell \\
\hline $\mathrm{L}_{\mathbf{M}}$ & $201 \mathrm{pH}$ & $804 \mathrm{pH} /$ Cell \\
\hline $\mathrm{C}_{\mathrm{MD}}$ & $295 \mathrm{fF}$ & $295 \mathrm{fF} /$ Cell \\
\hline $\mathbf{R}_{\mathrm{MD}}$ & $12 \Omega$ & $12 \Omega /$ Cell \\
\hline
\end{tabular}

theory [8]. As the microstrip line becomes longer and its dielectric substrate has a higher dielectric loss, a frequency-dependent dielectric loss must be considered in wideband modeling. A capacitor-resistor pair $\left(C_{M D}-\mathrm{R}_{M D}\right)$ model is used between the two metals, based on the Debye model to express the frequency-dependent dielectric loss [17].

And then, we obtained the parameter values (Table I) through the $S$-parameter fitting process using the measured $S$-parameter of a microstrip line without the through-hole signal via. The measured $S$-parameter was obtained by using the network analyzer (HP8753ES) from $1 \mathrm{MHz}$ to $3 \mathrm{GHz}$ and two commercial 4-cm 50- $\Omega$ coaxial cable assemblies (Tensolite, A Carlisle Corporation). The surface mount assembly (SMA) connector and shield of each coaxial cable assembly at one end were stripped off and each exposed signal line in $1 \mathrm{~mm}$ was directly soldered at each microstrip line end to reduce the mismatching effect. And each shield at the stripped end was soldered at the power plane and ground plane, respectively. In particular, this method is useful for measuring two microstrip lines located on both sides of test boards with separated reference planes but connected by the through-hole signal via without using conventional SMA connectors, which make large mismatching at the 


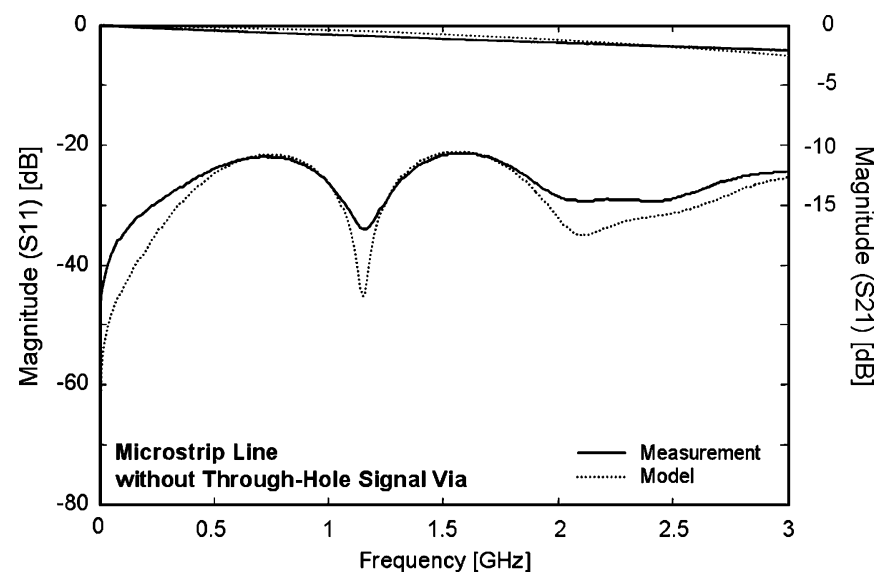

Fig. 6. S11 magnitudes (black and left scale) and S21 magnitudes (gray and right scale) of the balanced TLM microstrip line model (dotted line) and the measurement (solid line) of the microstrip line.

contact point of microstrip line, and a conventional prove station with micro size proves, which is used for measurement of single side patterned structure. From a capacitance and an inductance of Table I, we could confirm that the characteristic impedance of the designed microstrip line is $52 \Omega$. Fig. 6 shows the measured (solid line) and the calculated (dotted line) S11 magnitude (black and left scale) and S21 magnitude (gray and right scale) of the microstrip line. It is noted that the modeling and the measurement match each other closely. The major reason for the difference in the $S$-parameter comes from the limited frequency dependence of the loss model, which is described in the Debye model. In the Debye model, the first-order model was designed to use a single capacitor $\left(C_{M D}\right)$ and resistor $\left(R_{M D}\right)$ pair in series.

Fig. 5(b) shows the unit cell and the balanced TLM of the power/ground plane cavity. It has 18 circuit elements; eight inductors $\left(L_{P}\right)$ and eight resistors $\left(R_{P}\right)$ are used for the conduction current paths on the ground plane and on the power plane. A series capacitor $\left(C_{P D}\right)$ and resistor $\left(R_{P D}\right)$ pair is the Debye model. These parameters can be estimated from the (1), except for $R_{P D}$

$$
\begin{aligned}
C_{P D} & =\varepsilon_{r} \varepsilon_{0} \frac{l^{2}}{d}(F) \\
L_{P} & =\frac{\mu_{0} d}{4}(H) \\
R_{P} & =\frac{2}{\sigma_{c} t}(\Omega)
\end{aligned}
$$

where $\varepsilon_{r}$ and $\varepsilon_{0}$ are the relative permittivity and the permittivity of free space, respectively, $l$ is the length and width of the unit cell, $d$ is the distance between the power/ground plane, $\mu_{0}$ is the permeability of free space, and $\sigma_{\mathrm{c}}$ and $t$ are the conductivity and the thickness of copper, respectively.

Fig. 5(c) illustrates the unit cell of the power/ground plane cavity including the through-hole signal via and the via coupling model of the through-hole signal via part (inside dashed-line cylinder), which is combined with the balanced TLM of power/ ground plane cavity Fig. 5(b) as shown in Fig. 3. The balanced TLM of power/ground plane cavity is omitted in Fig. 5(c) to make the via coupling model clearer. Basically, the through-hole
TABLE II

PARAMETERS OF THE BALANCED TLM OF POWER/GROUND Plane CAVITY

\begin{tabular}{|c|c||c|}
\hline Notation & Value & Total Value \\
\hline $\mathbf{R}_{\mathbf{P}}$ & $0.5 \mathrm{~m} \Omega$ & $2 \mathrm{~m} \Omega /$ Cell \\
\hline $\mathbf{L}_{\mathbf{P}}$ & $314 \mathrm{pH}$ & $1256 \mathrm{pH} /$ Cell \\
\hline $\mathbf{C}_{\mathbf{P D}}$ & $226 \mathrm{fF}$ & $226 \mathrm{fF} /$ Cell \\
\hline $\mathbf{R}_{\mathbf{P D}}$ & $8 \Omega$ & $8 \Omega /$ Cell \\
\hline
\end{tabular}

TABLE III

PARAMETERS OF THE VIA COUPLING MOdEL

\begin{tabular}{|c|c||c|}
\hline Notation & Value & Total Value \\
\hline $\mathrm{L}_{\mathrm{N}}$ & $622 \mathrm{pH}$ & $1244 \mathrm{pH} /$ Cell \\
\hline $\mathrm{C}_{\mathrm{V}}$ & $123 \mathrm{fF}$ & $984 \mathrm{fF} /$ Cell \\
\hline $\mathrm{L}_{\mathrm{V}}$ & $752 \mathrm{pH}$ & $752 \mathrm{pH} /$ Cell \\
\hline
\end{tabular}

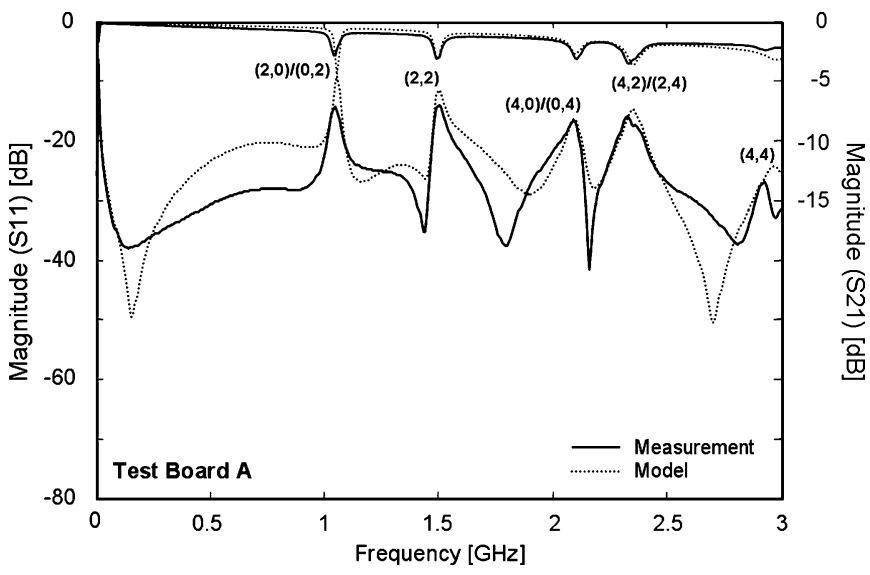

Fig. 7. S11 magnitudes (black and left scale) and S21 magnitudes (gray and right scale) of the proposed model (dotted line) and the measurement (solid line) of test board A. The numbers in round brackets are resonance mode numbers.

signal via model is a transmission line model of an unbalanced type scheme that is capacitively coupled to multiple reference plane edges $\left(C_{V}\right)$. Two $L_{N}$ is serially added to the model as the inductance to describe the high-impedance section between the end of the microstrip line and the via land pad, where the reference is absent because of via clearance. The metallic via drill hole is expressed by $L_{V}$.

With the models of the three unit cells, we composed the models of two test boards (Fig. 4). The extracted model parameters are listed in Tables II and III. The simulated and measured S11 and S21 magnitudes of test board A are shown in Fig. 7, and those of test board B are in Fig. 8. Both simulation and measurement show fair agreement of the resonance frequencies and associated modes depending on the via positions. The numbers in round brackets of the figures are the resonance mode numbers of the power/ground plane cavity. The resonance frequency of each mode number can be estimated with the following equation:

$$
\left(f_{r}\right)_{m n}=\frac{1}{2 \pi \sqrt{\mu \varepsilon_{r} \varepsilon_{0}}} \sqrt{\left(\frac{m \pi}{a}\right)^{2}+\left(\frac{n \pi}{b}\right)^{2}}
$$

where $f_{r}$ is the resonance frequency related to mode number $(m, n)$, and $a(14 \mathrm{~cm})$ and $b(14 \mathrm{~cm})$ are the width and length of 


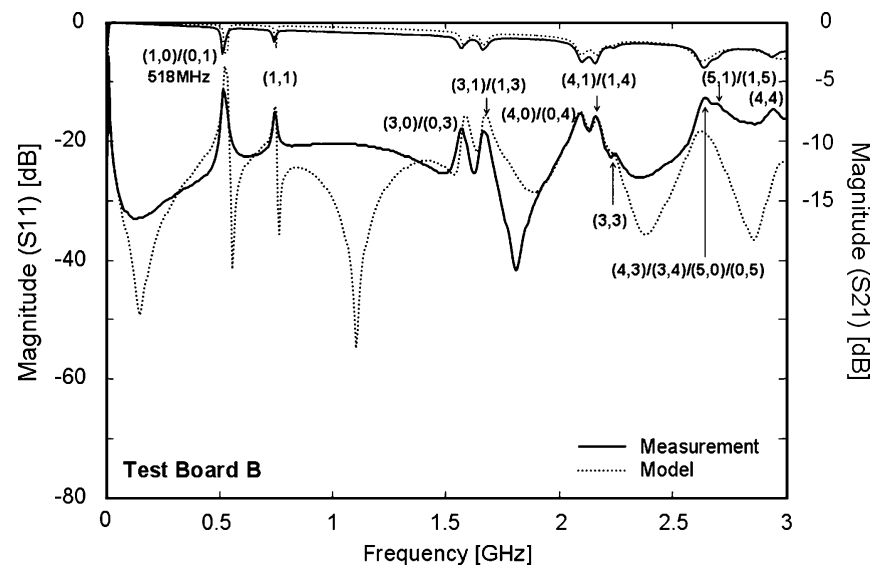

Fig. 8. S11 magnitudes (black and left scale) and S21 magnitudes (gray and right scale) of the proposed model (dotted line) and the measurement (solid line) of test board B. The numbers in round brackets are resonance mode numbers.

the power/ground plane cavity. The resonance frequencies are independent of the dielectric thickness. The slight discrepancy observed between the model and the measurement in Figs. 7 and 8 mainly comes from the limit of the frequency-dependent first-order loss Debye model used in the microstrip line model and the power/ground plane cavity model.

In the case of test board A, all the resonances of the "odd"numbered modes are considerably suppressed by positioning the via at the center of the power/ground plane. This is because of the mode properties and the open edge boundary condition of the rectangular shape. It means that the signal via does not suffer any high impedance of the ground return current path from the cavity resonance at the "odd"-numbered resonance frequencies. On the other hand, at the "even"-numbered resonance frequencies, the signal via has a very large amount of reflection loss in S11 and insertion loss in S21 due to cavity resonance. As a result, the high impedance produced by the power/ground plane cavity resonance imposes the high impedance at the return current path discontinuity, and the total input impedance at the feeding point of the microstrip line at port 1 in Fig. 3 becomes extremely high at the "even"-numbered mode resonances. Consequently, S11 magnitude increases and S21 magnitude decreases at the "even"-numbered mode resonances; that is, the return loss and the insertion loss increase. Furthermore, at the "even"-numbered mode resonances, a large amount of the field is developed at the power/ground plane edge and at the through-hole signal via, owing to the high impedance at the cavity edge generating cavity resonance. The excited cavity resonance is eventually propagated to the edge of the power/ground plane cavity, resulting in the radiated field emission from the cavity edge. The radiated field emission will be discussed in Section III. Most resonances are observed in case of test board B as shown in Fig. 8, but the "2"-numbered mode resonances disappear.

Fig. 9 shows the calculated impedance of the power/ground plane cavity at first four resoancnes. The relative power/ground plane cavity impedances are plotted with a relative scale from the white region for high impedance to the black region for low impedance. "A" and "B" in Fig. 9 are the positions of the through-hole signal vias in test board A and $\mathrm{B}$, respectively.
As mentioned previously, when the through-hole signal via is located in the high-impedance area (white area), it suffers significant return loss and insertion loss at the signal line. On the other hand, when the through-hole signal via is located in the low-impedance area (black area), the resonances disappear from the $S$-parameters, avoiding the signal return loss and insertion loss. Therefore, the $(1,0) /(0,1)$ mode and the $(1,1)$ mode are not shown in Fig. 7 , and the $(2,0) /(0,2)$ mode and the $(2,2)$ mode are not shown in Fig. 8. Other resonances, whose impedances are not plotted in Fig. 9, can be analyzed analogously. Hence, the signal loss is heavily dependent not only on the resonance mode, but also on the position of the via. Consequently, the via position should be carefully treated to minimize the signal loss and the radiated field emission problem from the power/ground plane cavity edge.

\section{RAdiated FIELD EMISSION From POWER/GROUND Plane Cavity EdGE}

As discussed in Section II, increased insertion loss is observed at the power/ground plane cavity resonance frequencies when the microstrip lines exchange the reference planes through the through-hole signal via. A voltage is induced across the power/ground cavity by the signal return current of the throughhole signal via. As a result, a power/ground voltage fluctuation is generated and propagated toward the edge of the power/ground plane cavity, producing standing waves in the cavity at the resonance frequencies. Consequently, the standing electric waves are distributed with the same shape as the impedance curve, as shown in Fig. 9, and produce maximum magnetic current at the open edges of the power/ground plane cavity. As a result, the magnetic current at the open boundary of the power/ground plane cavity edge becomes the source of the radiated field emission. This is the mechanism of the radiated field emission from the power/ground plane cavity edge excited by the through-hole signal via. As mentioned previously, the excited resonance frequencies are dependent on the location of the via as well as the cavity size. By using the proposed balanced TLM model, we can calculate the voltage and magnetic current distribution at the power/ground cavity edge. Subsequently, the radiated field emission can be deduced from the calculated magnetic current.

Fig. 10 shows the calculation procedure for the radiated field emission with the voltage distribution obtained from the proposed model. E-field $(E)$ at the power/ground plane cavity edge is calculated by

$$
\vec{E}=\frac{\vec{V}}{d}
$$

where $V$ is the calculated voltage of each unit cell at the power/ ground plane edge, and $d$ is the dielectric thickness of the power/ ground plane. The magnetic current $(M)$ is calculated by using Huygens' Principle of the aperture structure [9] as

$$
\vec{M}=-2 \vec{n} \times \vec{E}
$$

where $n$ is the normal unit vector on the surface of the power/ ground plane cavity edge, as shown in Fig. 10. The vector po- 


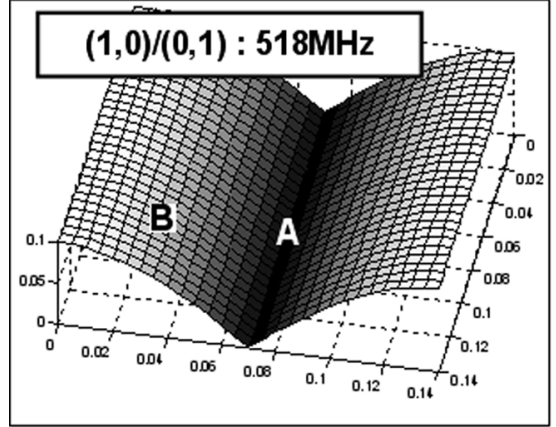

(a)

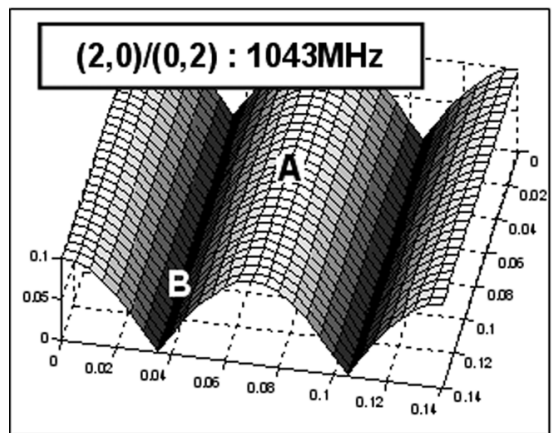

(c)

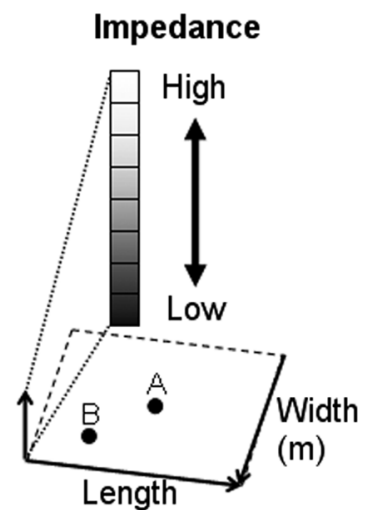

(m)
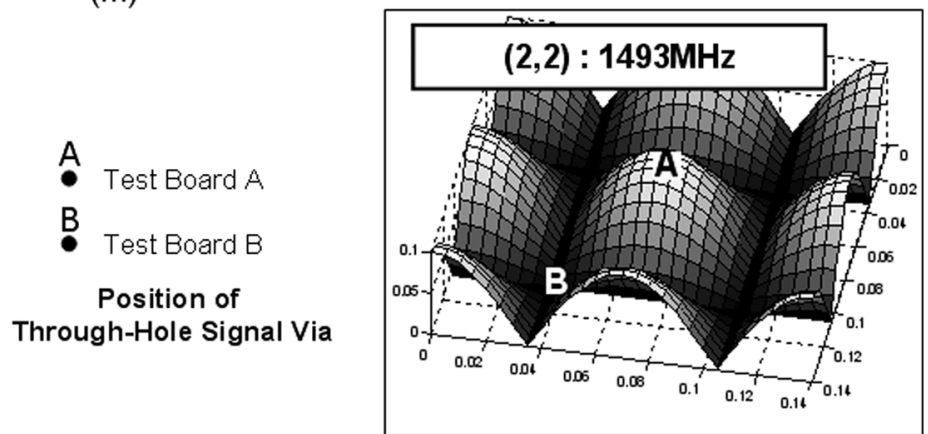

(d)

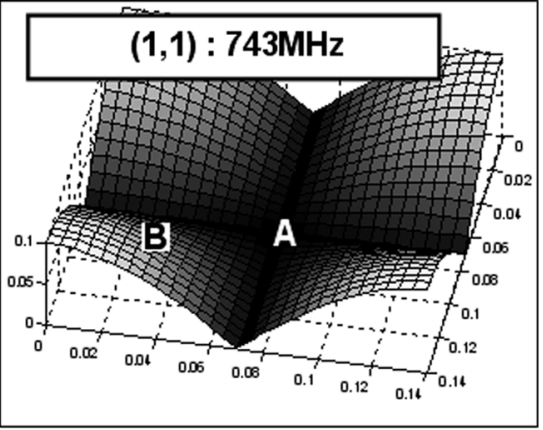

(b)

Fig. 9. Impedance distributions of test boards with the open-ended power/ground plane cavity at the first four resonances.

tential $(F)$ is obtained with $M$, and finally the radiated electric field $\left(E_{\text {rad }}\right)$ and magnetic field $\left(H_{\text {rad }}\right)$ are calculated from

$$
\begin{aligned}
\vec{F} & =\frac{\varepsilon}{4 \pi} \int_{l} \vec{M} \frac{e^{-j k R}}{R} \cdot d l \\
\vec{E}_{\mathrm{rad}} & =-\frac{1}{\varepsilon} \nabla \times \vec{F} \\
\vec{H}_{\mathrm{rad}} & =-\frac{1}{j 2 \pi f \mu} \nabla \times \vec{E}_{\mathrm{rad}}
\end{aligned}
$$

where $\varepsilon, \mu$, and $k$ are the permittivity, permeability, and propagation constant in free space, respectively, $l$ is the length of the magnetic current dipole, $R$ is the distance between $M$ and the field measurement points, " $\nabla \times$ " is the curl of the vector, and $f$ is the frequency. The final radiated field emission from the power/ground plane cavity edge at a measuring point is calculated from

$$
\begin{aligned}
& E_{\mathrm{rad} \Phi}=\sum_{m=1}^{q} E_{\mathrm{rad} \Phi}\left(R_{m}\right) \\
& H_{\mathrm{radZ}}=\sum_{m=1}^{q} H_{\mathrm{radZ}}\left(R_{m}\right)
\end{aligned}
$$

where

$$
\begin{aligned}
F_{Z}\left(R_{m}\right) & =\frac{\varepsilon_{0} M d l}{4 \pi R_{m}} e^{-j k R_{m}} \cdot a_{Z} \\
E_{\mathrm{rad} \Phi}\left(R_{m}\right) & =\frac{M d l}{4 \pi} \frac{\left(1+j k R_{m}\right)}{R_{m}^{2}} e^{-j k R_{m}} \cdot a_{\Phi} \\
H_{\mathrm{radZ}}\left(R_{m}\right) & =\frac{M d l}{j 4 \pi k \mu_{0} c} \frac{\left(1+j k R_{m}-k^{2} R_{m}^{2}\right)}{R_{m}^{2}} e^{-j k R_{m}} \cdot a_{Z}
\end{aligned}
$$

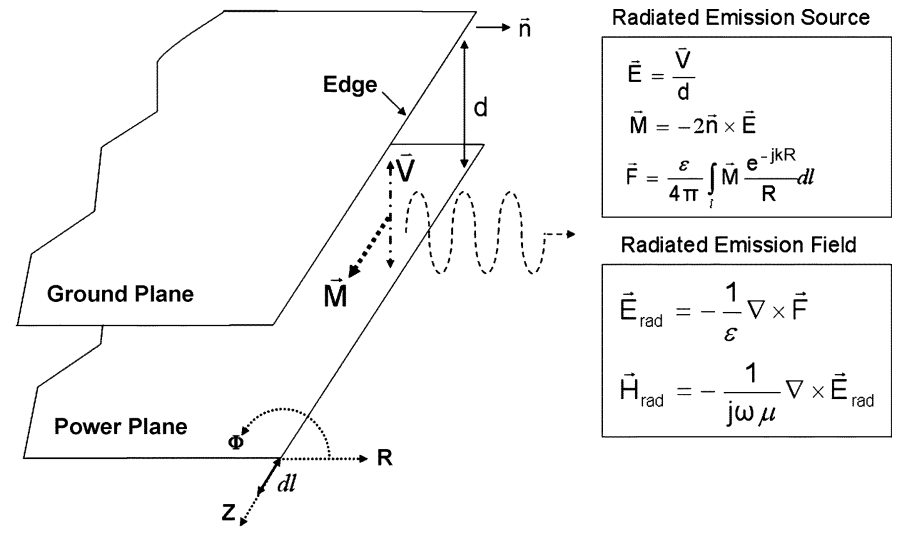

Fig. 10. Calculation of the radiated field emission from the power/ground plane cavity edge based on Huygens' Principle [6]. The coordinate is the cylindrical one $(R, \Phi, Z)$, whose $Z$-direction is located on the power/ground plane cavity edge.

and components of the other direction fields are "zero." The cylindrical coordinate used in the equations is shown in Fig. 10 as $R, \Phi$, and $Z, q$ is the number of unit cells at the power/ground plane edge, $R_{m}$ is the distance from each unit cell to the single field calculation point, $d l$ is the length of the unit cell, and " $a_{()}$" with coordinates is the unit vector in each direction.

To verify the calculated radiated field emission from the proposed model of the test boards, we measured the radiated field emissions from the power/ground plane cavity edge of test board A and test board B of Fig. 4. The near magnetic field was measured using a loop antenna (4-cm diameter) and a network analyzer (HP8753ES), which was scanned from $1 \mathrm{MHz}$ to $3 \mathrm{GHz}$, while the field emission was excited by the through-hole signal 
via at the test boards. The top microstrip line (port 1 in Fig. 3) of the test board was connected to the forward port of the network analyzer, and the bottom microstrip line (port 2 in Fig. 3) was terminated with a $50-\Omega$ load. The loop antenna was connected to the reverse port of the network analyzer. The center of the loop antenna was fixed $3 \mathrm{~cm}$ from the middle of the power/ground plane cavity edge. The area of the loop antenna was vertically oriented to both the test board edge surface and the test board surface, so that the loop antenna could measure the $Z$-component of the magnetic field emission. Thus, the loop antenna could measure the magnetic field in the $Z$-direction made by the magnetic current of $Z$-direction $(M)$ at the test board edge. The measured radiated field emission does not include the radiated emission from the microstrip lines, since the loop antenna does not detect the magnetic field in the $\Phi$-direction, which is the main magnetic field component of the microstrip lines.

To confirm the suppression ability of measurement setup against the direct radiated emission from the microstrip lines, we have measured the noise level and the test board without through-hole signal via. Both measured results remain below $-80 \mathrm{~dB}$. Therefore, we could say that the following measured radiated emissions show the only radiated emissions from the power/ground plane edges of the test boards. Moreover, we could say that a power/ground plane edge radiation is induced by the only role of a through-hole signal via in the test boards.

Fig. 11 shows the calculated radiated field emission (dotted line) from the proposed model of the test boards, and the measured one (solid line). Fig. 11(a) shows the radiated field emission of test board A, while Fig. 11(b) shows that of test board B. The proposed model closely predicts the radiated field emissions from the power/ground plane cavity edge excited by the through-hole signal via. The difference between the measurement and the calculation in the low-frequency range arises from omission of the loop antenna gain in the calculation. The loop antenna gain in the low-frequency range is lower than that in the high-frequency range. The peaks of radiated field emission occur at resonances of the test boards and their levels are larger by over $30 \mathrm{~dB}$ than the noise level $-80 \mathrm{~dB}$. The numbers in round brackets appear at the same frequencies as those of Figs. 7 and 8 . This means that the radiated field emission is strongly correlated with the increased insertion loss of the microstrip line. Since the insertion loss is increased maximally owing to the through-hole signal via, related to the high impedance of the power/ground plane cavity, it can be assumed that the lost signal power, shown as under-peaks in the insertion loss, remains in the power/ground plane cavity. The power continuously injected into the cavity is emitted through the open edge. Therefore, the radiated field emission is maximized at the resonance of the power/ground plane cavity, where the insertion loss of the microstrip line with the through-hole signal via is also maximized.

The proposed mechanism for the radiated field emission excited by the through-hole signal via is verified from the measurement and the calculation of the radiated field emissions. This is significant for high-performance system design, because the conventional planar-type shielding method applied to the high-frequency signal, such as a strip line, is no longer the safe solution for the radiated field emission problem. The planar

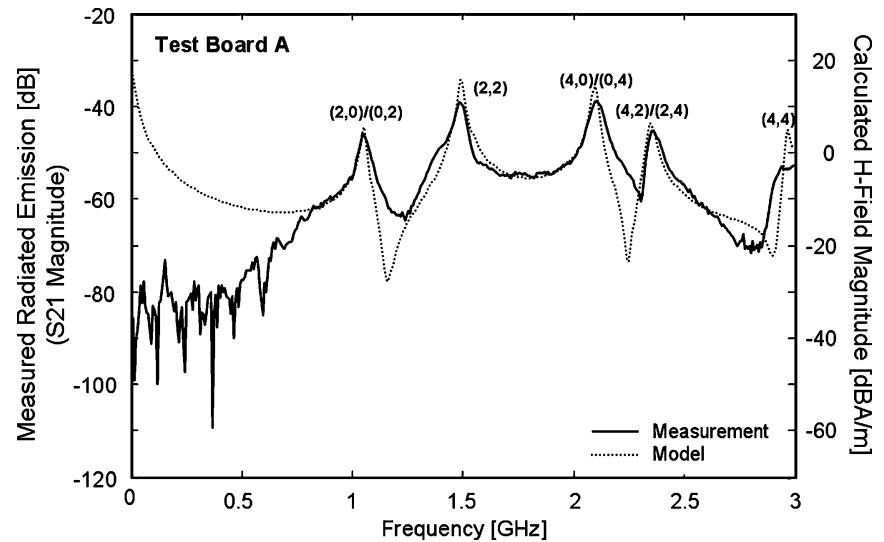

(a)

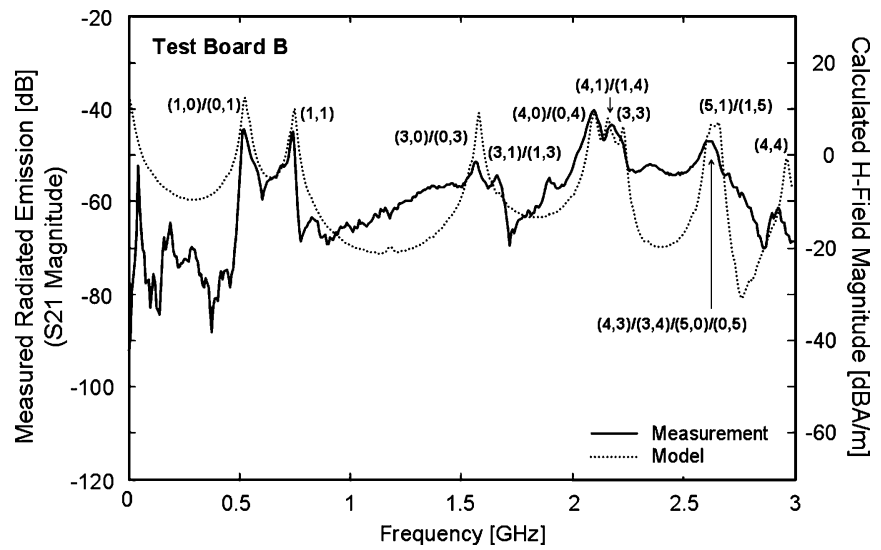

(b)

Fig. 11. (a) Measured (solid line and left scale) and calculated (dotted line and right scale) radiated field emissions from the power/ground plane edge of test board A. The calculation does not include the receiving antenna gain. Numbers in round brackets are resonance mode numbers. (b) Measured (solid line and left scale) and calculated (dotted line and right scale) radiated field emissions from the power/ground plane edge of test board B.

shielding method always includes the via, producing the cavity that produces the new radiated field emission from the cavity edge. Moreover, since the cavity is the power storage tank, the problem becomes more serious. Therefore, the signal via must be designed following investigation of the high impedance of the cavity, which can be suppressed by selecting its position as shown in Figs. 7, 8, and 11. It is necessary to simulate the situation mixed with signal integrity and power integrity to predict the radiated field emissions accurately. The proposed balanced TLM and via coupling model is useful for efficiently analyzing various problems arising from mixed signal integrity (SI), power integrity (PI), and EMI without regard to system structure.

\section{EfFect of a Decoupling CAPacitor Fence ON RADIATED FIELD EMISSION}

There are several ways to suppress the radiated field emission from the PCB edge. Some of the methods were discussed in the previous section. Another method of diminishing the emission is to lessen the power/ground plane impedance at the signal via or at the power/ground plane cavity edge. A thinner dielectric 


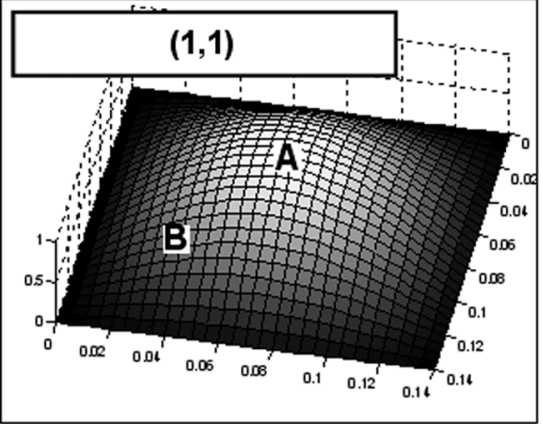

(a)

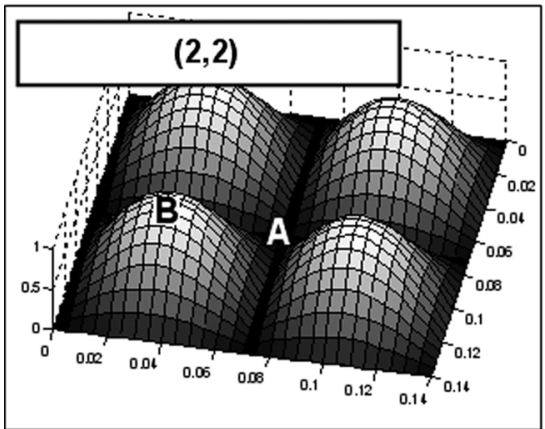

(c)

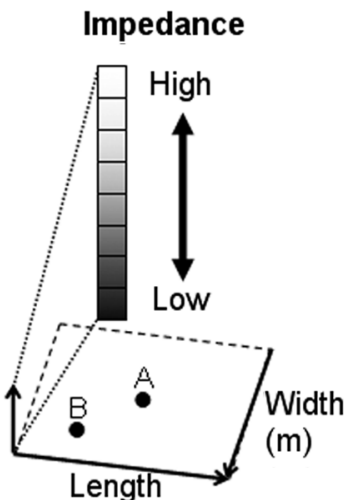

(m)

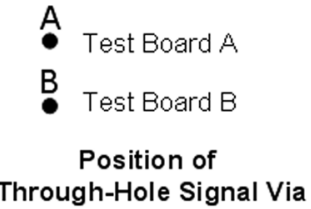

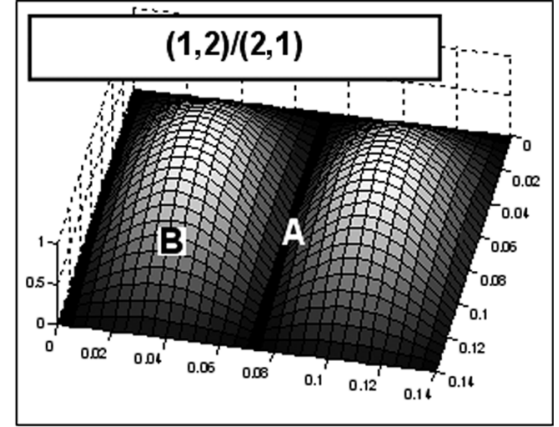

(b)

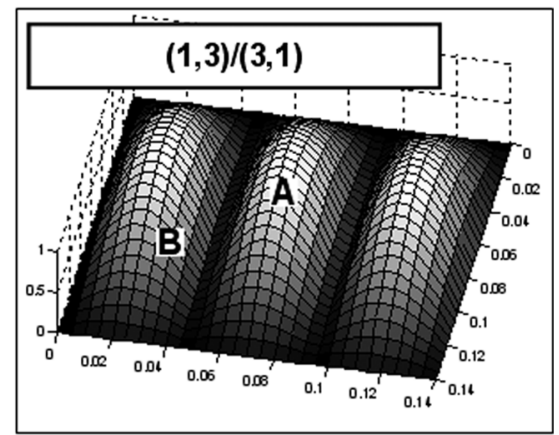

(d)

Fig. 12. Impedance distributions of test boards with the short-ended power/ground plane cavity at the first four resonances.

insulator board can be used for this purpose. In addition, decoupling capacitors can be placed near the via or at the cavity edge. These decoupling capacitors provide a low-impedance return current path near the via, suppressing the noise voltage generation, or they produce a low-impedance boundary condition and a low-voltage fluctuation at the cavity edge. However, since the signal via is usually adopted for convenient routing in high-density board, it is not always feasible to locate the signal via freely in a desirable position. Moreover, it is not easy to find an area near the via to place a considerable number of decoupling capacitors on the densely populated board. If the decoupling capacitors are widely scattered on the board, the decoupling effect is diminished by the increased path inductance in the hundreds of magahertz range.

The decoupling capacitor fence [De-Cap Fence; Fig. 13(a)] at the power/ground plane cavity edge is one feasible way of reducing the radiated field emission. Since the De-Cap Fence is a line formation, the necessary component space can be minimal, and the equivalent series inductance (ESL) can be reduced by the parallel connection. As the decoupling capacitors are located tightly, the ESL can be more effectively reduced to accomplish the low-impedance boundary condition at the cavity edge. The cavity edge becomes nearly a short boundary, which generates a small voltage and consequently small radiated field emissions at the cavity edge. Since the edge boundary condition is altered to the short boundary condition by the De-Cap Fence, resonance frequencies, the field distribution, and the impedance profile at the power/ground plane cavity (Fig. 12) are quite different from those of the open boundary cavity edge (Fig. 9). We have observed this in the following simulations and measure- ments. Furthermore, we have successfully analyzed the observation that the resonance frequencies are shifted to higher frequencies as the number of decoupling capacitors is increased.

In this paper, we have studied the effect of the De-Cap Fence based on measurement and on the balanced TLM method. The procedure for the measurement and analysis is identical to that discussed in the previous section. The test boards have the same structures of Fig. 4 except for their De-Cap Fences. Two different pitches of the decoupling capacitors were considered: a 1 ea/5-mm De-Cap Fence and a 1 ea/10-mm De-Cap Fence. The decoupling capacitor numbers of two pitch De-Cap Fences are 25 ea/edge and 13 ea/edge, respectively. As a result, four different test boards were simulated and measured in this section. To conduct the balanced TLM simulation, we have proposed the model of the decoupling capacitor and the power/ground via as presented in Fig. 13(b). The decoupling capacitor has $10 \mathrm{nF}, 330 \mathrm{pH}$ ESL (ESL1), and 0603 size. ESL2 (power via inductance) and $4 C_{V}$ (via clearance capacitance) are added by using that of the through-hole signal via model in Section II. The 10-nF capacitor is selected to avoid overlapping the self-resonance frequency of the decoupling capacitors onto the power/ ground plane cavity resonance frequencies. The self-resonance frequency of the decoupling capacitor is lowered to $50 \mathrm{MHz}$ when combined with the power via parasitics.

Fig. 14(a) shows the measured (solid line) and the calculated (dotted line) radiated field emissions from test board A with the 1 ea/5-mm De-Cap Fence. Since the De-Cap Fence provides nearly a short boundary, the field distribution are distinctly different from those in Fig. 11(a), and all the "even"-numbered mode resonances have disappeared. This means that the spec- 


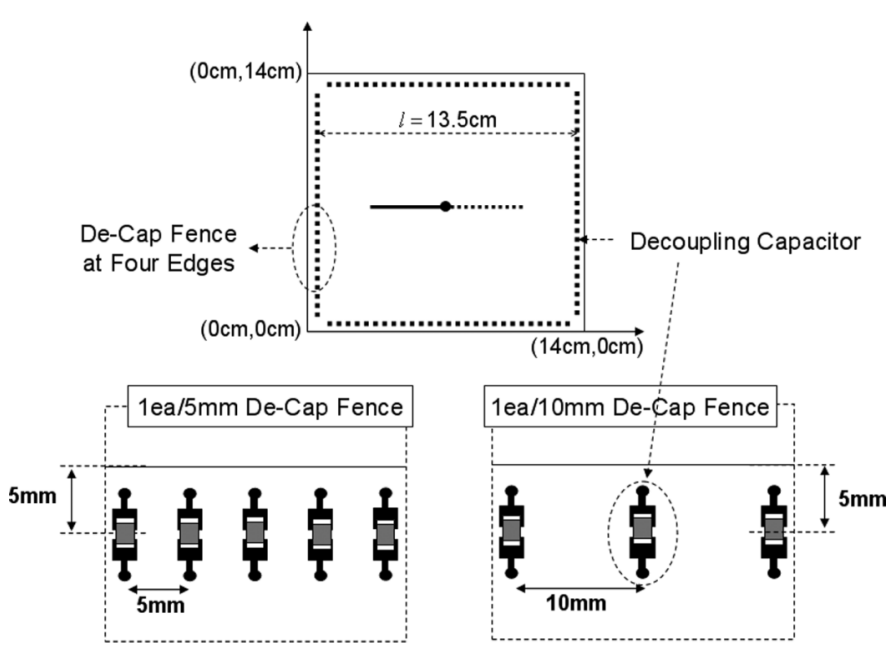

(a)

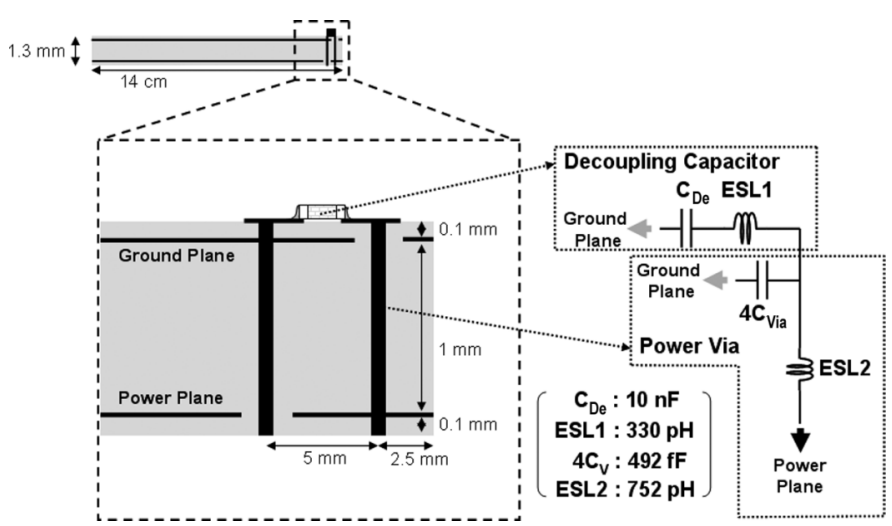

(b)

Fig. 13. (a) Structures of De-Cap Fence, which have decoupling capacitors at the power/ground plane edge. Two types $(1 \mathrm{ea} / 5 \mathrm{~mm}$ and $1 \mathrm{ea} / 10 \mathrm{~mm})$ are applied to both test boards A and B. (b) Decoupling capacitor and its power via model for simulation of the De-Cap Fence embedded into the proposed model of test boards A and B.

trum of the radiated field emission is altered by the boundary condition. In addition, since the De-cap fence does not guarantee the perfect short boundary condition, noise voltage remains at the cavity edge, producing radiated field emission. The ESL of De-Cap Fence becomes a secondary source of the radiated field emission, but the amount of the radiated field emission is smaller. Fig. 14(b) presents the measured (solid line) and the calculated (dotted line) radiated field emissions from test board B with the $1 \mathrm{ea} / 5-\mathrm{mm}$ De-Cap Fence. On comparing with Fig. 11(b), some of the "even"-numbered resonance modes appear, while all " 0 "- and "4"-numbered modes are diminished. Fig. 14(c) shows the measured (solid line) and the calculated (dotted line) radiated field emissions from test board A with the 1 ea/10-mm De-Cap Fence. By comparing it with Fig. 14(a), it is found that all resonance frequencies are moved toward lower frequencies, while the mode numbers remain identical. Similar frequency shifts are observed between Fig. 14(b) and 14(d).

The frequency shift can be explained by the short stub effect of the ESL of the De-Cap Fence (Fig. 15). Since the effective ESL $\left(L^{\prime}\right)$ is present at the De-Cap Fence, the electrical length of the cavity $\left(l_{\text {eff }}=l+2 l_{\text {stub }}\right)$ is longer than the physical length $(l)$ of the power/ground plane cavity. Since the effective ESL is dependent on the pitch of the decoupling capacitors in the De-Cap Fence, the effective electrical length of the cavity changes as the pitch of De-Cap Fence varies. As the pitch becomes smaller, the effective ESL and the electrical length of the cavity become lower resulting in higher resonance frequencies. In Fig. 14(a) and (c), the effective electrical lengths of the power/ground plane cavity are 13.9 and $14.9 \mathrm{~cm}$ at each $(1,1)$ mode $(734,683 \mathrm{MHz})$, respectively. These are explained by the following relations:

$$
\begin{aligned}
l_{\text {eff }} & =l+2 l_{\text {stub }}=\frac{c}{\sqrt{2 \varepsilon_{r}} \cdot f_{r}} \\
L^{\prime} & =\frac{Z_{P 0}}{4 \pi f_{r}} \tan \left(\frac{\pi\left(2 l_{\text {stub }}\right)}{l_{\text {eff }}}\right) \\
L^{\prime} \cong & \frac{(\mathrm{ESL} 1+\mathrm{ESL} 2)}{\mathrm{N}}
\end{aligned}
$$

where $f_{r}$ is the $(m, n)$ mode resonance frequency of the cavity with the De-Cap Fence, $Z_{P O}$ is the plane impedance, $l$ is the physical distance from the De-Cap Fence at one end of the test board to the De-Cap Fence at the other end $(l=13.5 \mathrm{~cm}), l_{\text {stub }}$ is the added electrical stub length to the physical cavity size, and $N$ is the number of decoupling capacitors per edge [19].

Equations (8)-(10) are developed using the following arguments. When standing waves at the resonance frequencies are developed, the voltage minimum positions of the standing waves does not appear at the physical power/ground plane cavity edge owing to the finite effective ESL of the De-Cap Fence $\left(L^{\prime}\right)$. Hence, the standing waves are extended outside the cavity by the extra electrical stub length $\left(2 l_{\text {stub }}\right)$. Fig. $15(\mathrm{a})$ and (b) describe these concepts. Thus, (9) is derived from

$$
\begin{aligned}
j 4 \pi f_{r} L^{\prime} & =Z_{P 0} \frac{0_{(\text {short })}+j Z_{P 0} \tan \left\{k_{r}\left(2 l_{\text {stub }}\right)\right\}}{Z_{P 0}+j 0_{(\text {short })} \times \tan \left\{k_{r}\left(2 l_{\text {stub }}\right)\right\}} \\
& =j Z_{P 0} \tan \left\{k_{r}\left(2 l_{\text {stub }}\right)\right\}
\end{aligned}
$$

where

$$
\begin{aligned}
k_{r} & =\frac{2 \pi}{\lambda_{r}}=\frac{2 \pi}{2\left(l+2 l_{\mathrm{stub}}\right)}=\frac{\pi}{l_{\mathrm{eff}}} \\
Z_{P 0} & =\sqrt{\frac{L_{\text {plane }}}{C_{\text {plane }}}} .
\end{aligned}
$$

In (11), $0_{\text {(short) }}$ indicates the perfect short termination at the end of the electrical stub, $k_{r}$ is the propagation constant determined by (8), which comes from the relation that $f_{r}$ is $\sqrt{ } 2$ times the $(1,0)$ mode resonance frequency, and (13) is determined from the inductance and capacitance of the power/ground plane cavity.

As shown in Fig. 14(a), the $(1,1)$ mode resonance frequency $f_{r}$ is $734 \mathrm{MHz}, Z_{P O}$ is $1.4 \Omega, l$ is $13.5 \mathrm{~cm}$, and $l_{\text {stub }}$ is $0.2 \mathrm{~cm}$, leading to the $L^{\prime}$ of the $1 \mathrm{ea} / 10 \mathrm{~mm}$ De-Cap Fence of $14 \mathrm{pH} /$ edge. Analogously, the $L^{\prime}$ of the $1 \mathrm{ea} / 5 \mathrm{~mm}$ De-Cap Fence is $49 \mathrm{pH} /$ edge, where the $f_{r}$ is $683 \mathrm{MHz}$ and $l_{\text {stub }}$ is $0.7 \mathrm{~cm}$ as shown in Fig. 14(c). As expected, the narrowly spaced De-Cap Fence considerably lowers the effective ESL, and moves the resonance frequencies toward lower frequencies.

We can also evaluate the suppression efficiency of the De-cap Fence by comparing Fig. 14 with Fig. 11. For convenience in 


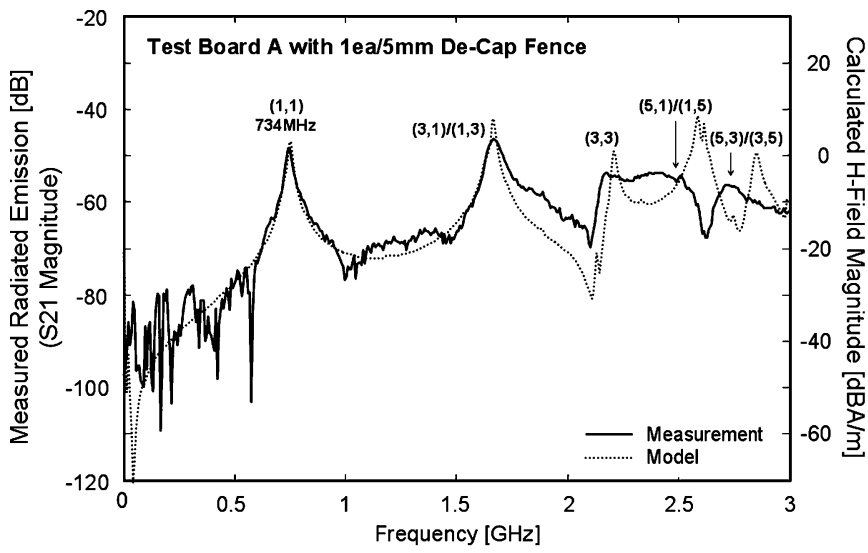

(a)

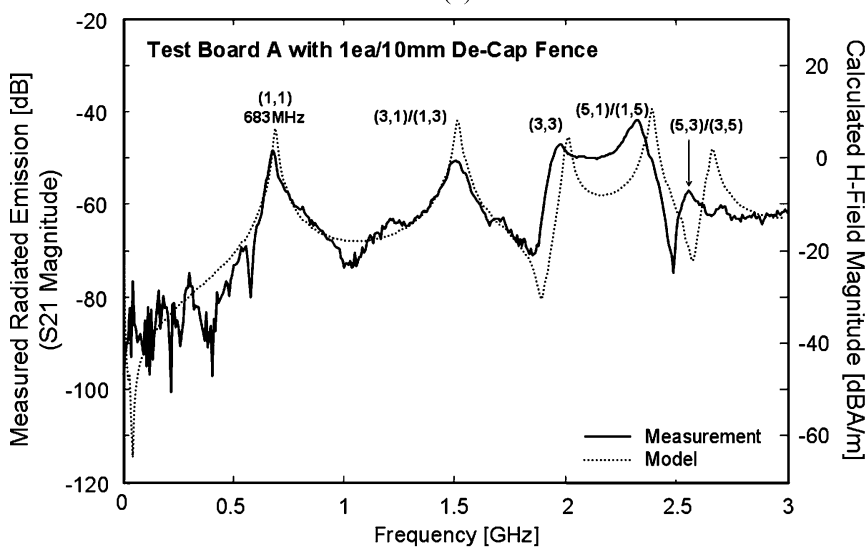

(c)

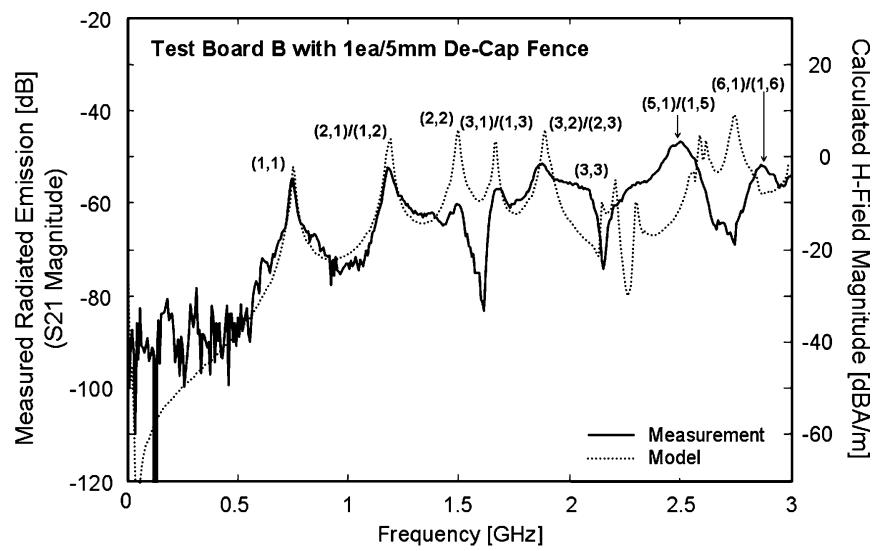

(b)

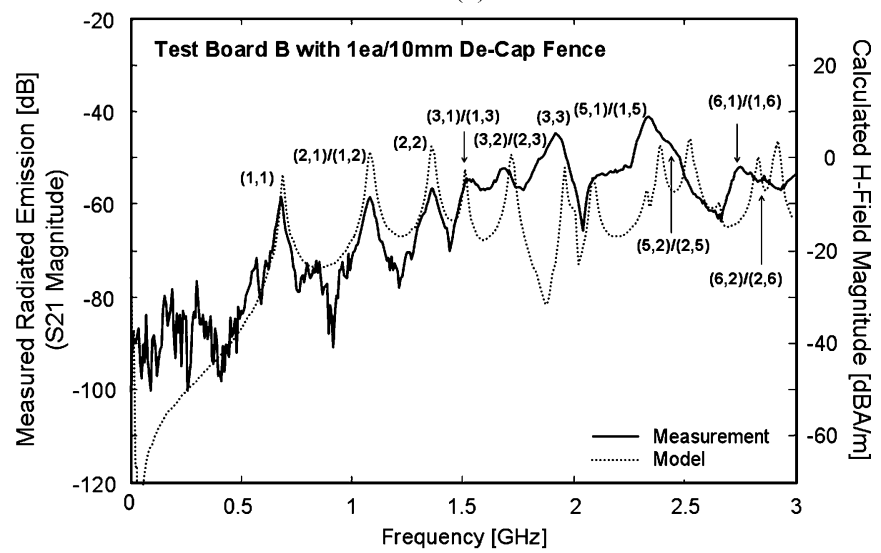

(d)

Fig. 14. (a) Measured (solid line and left scale) and calculated (dotted line and right scale) radiated field emissions from the power/ground plane edge of test board A with 1 ea/5-mm De-Cap. (b) Measured (solid line and left scale) and calculated (dotted line and right scale) radiated field emissions from the power/ground plane edge of test board B with 1 ea/5-mm De-Cap Fence. (c) Measured (solid line and left scale) and calculated (dotted line and right scale) radiated field emissions from the power/ground plane edge of test board A with 1 ea/10-mm De-Cap Fence. (d) Measured (solid line and left scale) and calculated (dotted line and right scale) radiated field emissions from the power/ground plane edge of test board B with 1 ea/10-mm De-Cap Fence.

comparison, the peak values of the measured radiated field emissions for each case are selected and compared. In test board type A, the 1 ea/5-mm De-Cap Fence suppresses the radiated field emission by $7 \mathrm{~dB}$, comparing the value at $f=734 \mathrm{MHz}$ of $(1,1)$ mode in Fig. 14(a) and the value at $f=1493 \mathrm{MHz}$ of $(2,2)$ mode in Fig. 11(a). The 1 ea/10-mm De-Cap Fence suppresses the emission by $3.5 \mathrm{~dB}$ in considering with the value at $f=2513 \mathrm{MHz}$ of $(5,1) /(1,5)$ mode in Fig. 14(c). In test board type B, the 1 ea/5-mm De-Cap Fence achieves 6-dB reduction at $f=2513 \mathrm{MHz}$ of $(5,1) /(1,5)$ mode of Fig. 14(b) compared with the value at $f=2133 \mathrm{MHz}$ of $(4,0) /(0,4)$ mode in Fig. 11(b). The 1 ea/10-mm De-Cap Fence achieves 1-dB reduction at $f=2513 \mathrm{MHz}$ of $(5,1) /(1,5)$ mode. Consequently, it is confirmed that the suppression efficiency is enhanced by using a densely pitched De-Cap Fence, which leads to a less inductive short boundary at the power/ground cavity edge, resulting in smaller induced voltage fluctuation at the power/ground cavity edge. This suppression effect is further enhanced by using a special decoupling capacitor with an extremely low ESL. Furthermore, the suppression effect can be improved by using a thinner dielectric thickness power/ground plane cavity, which reduces the ESL of the power/ground via.
In this study of the De-Cap Fence, the proposed model is again found to be an efficient method for predicting the radiated field emission from the power/ground plane cavity edge, where the power/ground plane cavity contains a number of passive devices and complex structures. The proposed model also predicts the De-Cap Fence effects. However, at the higher frequency, the difference between the model and the measurement becomes evident. This arises from errors in high-frequency loss of the power/ground plane model, as mentioned in previous sections, and inaccuracy of the decoupling capacitor model, which has a single series-resonance frequency at $50 \mathrm{MHz}$, while multiple series and parallel resonances may cover gigahertz frequency ranges. As shown in the ESL effects of the decoupling capacitor and the power/ground via, the accuracy of the simulation relies on the reliability of the preciseness of the component models. Moreover, at very high frequency, the frequency-dependent loss model is crucial to maintain correct simulation results.

\section{CONCLUSION}

In this paper, the balanced TLM and the via coupling model are proposed for an efficient simulation of the radiated field emission problem from the power/ground plane cavity edge 


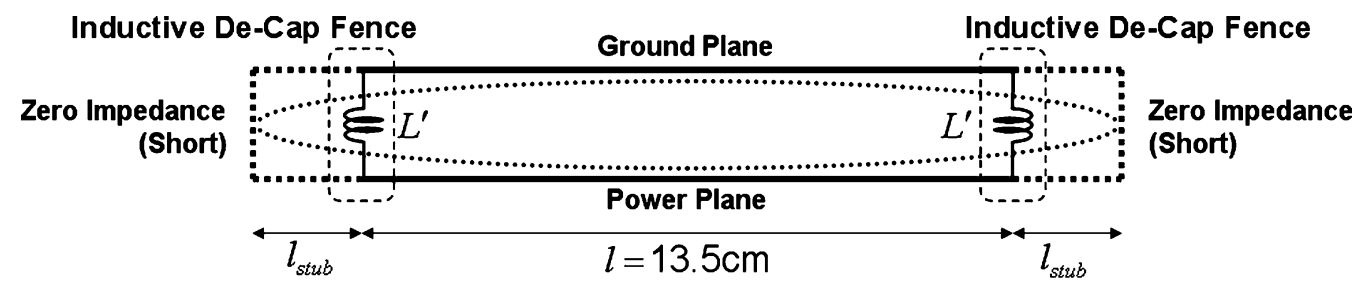

(a)

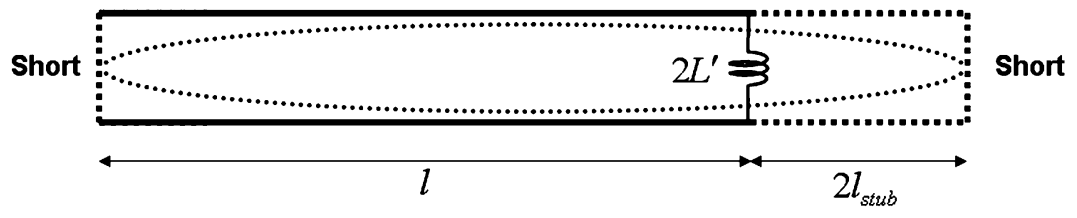

(b)

Fig. 15. Estimation of resonance frequency shift with different inductive termination effects of the De-Cap Fence $\left(L^{\prime}\right)$ as shown in Fig. 14 .

when the radiated field emission is excited by the through-hole signal via in the multilayer package and PCB. The proposed balanced TLM is a new TLM method having equivalent circuit model elements at the signal path as well as at the return current path in a balanced manner. The conventional TLM cannot be used for some two-dimensional analysis cases, where the impedance of the ground return current is not negligible. Such cases occur when there are slots or cutouts in the ground plane, producing high-impedance sections of the ground return current path, especially when the signal trace is switching the reference planes through the signal via in the multilayer stack, in which case the ground return current is disrupted at the via, and the ground impedance becomes extremely high at the resonance frequencies of the power/ground plane cavity. The proposed balanced TLM is also useful when there is an ambiguity of the ground reference point assignment if the multilayer board has multiple ground planes in a stack. It is also applicable to any structure of the power/ground plane, even including diverse line structures and discrete devices. It is also compatible with any spice-type circuit simulator.

To verify the proposed model, we have simulated and measured the radiated field emission with a series of test boards. The simulation agrees fairly well with the measurement, which confirms the preciseness and usefulness of the proposed model. It is also shown that the through-hole signal via is a considerable source of signal loss as well as radiated field emission. The radiated field emission from the power/ground plane cavity edge is the result of the return current discontinuity at the through-hole signal via. The return current discontinuity is caused by the high impedance of the power/ground plane cavity resonance. As a result, the insertion loss and the return loss of the microstrip line, including the through-hole signal via, are considerably increased at the cavity resonances. The return current discontinuity induces voltage fluctuations at the power/ground plane cavity. The induced voltage fluctuations propagate toward the power/ground plane cavity edge and build up standing waves by reflection at the cavity edge at the resonance frequencies, inducing the magnetic current at the open edge of the power/ ground plane cavity. The magnetic current becomes the source of radiated field emission. From the analysis, it is asserted that the resonance effect can be suppressed by controlling the position of the through-hole signal via.

The proposed model was also applied to the analysis of the De-Cap Fence, which is used for suppressing the radiated field emission by offering a low-impedance boundary condition at the power/ground plane cavity edge. In this application, the proposed model closely predicts its performance. It is confirmed that the effective inductance of the De-Cap Fence is a crucial factor, which is controlled by the space between decoupling capacitors and the ESLs of the decoupling capacitor and the power/ground via. As predicted by the proposed model, the De-Cap Fence changes the resonance mode and frequency of the plane cavity, and reduces the radiated field emission by $7 \mathrm{~dB}$. The suppression effect can be further enhanced by increasing the number of decoupling capacitors, choosing decoupling capacitors of low ESL, and reducing the board dielectric thickness.

\section{REFERENCES}

[1] D. G. Kam, H. Lee, J. Kim, and J. Kim, “A new twisted differential line structure on high-speed printed circuit boards to enhance immunity to crosstalk and external noise," IEEE Microw. Wireless Compon. Lett., vol. 13, no. 9, pp. 411-413, Sep. 2003.

[2] J. Kim, H. Kim, and J. Kim, "Efficient on-chip decoupling capacitor design on an 8-bit microcontroller to reduce simultaneous switching noise and electromagnetic radiated emission," IEICE Trans. Commun., vol. E86-B, no. 6, pp. 2077-2080, Jun. 2003.

[3] J. P. Simpson, R. R. Goulette, and G. I. Costache, "Radiation from microstrip transmission lines," in Proc. IEEE Int. Symp. Electromagn. Compat., Seattle, WA, Aug. 1988, pp. 340-343.

[4] D. M. Hockanson, J. L. Drewniak, T. H. Hubing, T. P. Van Doren, F. Sha, and M. J. Wilhelm, "Investigation of fundamental EMI source mechanisms driving common-mode radiation from printed circuit boards with attached cables," IEEE Trans. Electromagn. Compat., vol. 38, no. 4, pp. 557-565, Nov. 1996.

[5] S. Ahn, J. Kim, H. Lee, J.-G. Byun, C.-S. Choi, H.-J. Hwang, and J. Kim, "Experimental investigation of radiated emission from flexible printed circuit (FPC) cable," in Proc. IEEE Int. Symp. Electromagn. Compat., Minneapolis, MN, Aug. 2002, pp. 883-888.

[6] N. J. Ryan, D. A. Stone, and B. Chambers, "Application of the FD-TD method to modeling the electromagnetic radiation from heatsinks," in Proc. 1997 10th Int. Conf. Electromagn. Compat., Warwick, U.K., 1997, pp. 119-124.

[7] S. Radu and D. Hockanson, "An investigation of PCB radiated emissions from simultaneous switching noise," in Proc. IEEE Int. Symp. Electromagn. Compat., Seattle, WA, Aug. 1999, pp. 893-898. 
[8] D. M. Pozar, Microwave Engineering. Reading, MA: Addison-Wesley, Inc., 1990.

[9] C. A. Balanis, Antenna Theory: Analysis and Design. New York: Wiley, 1997.

[10] M. Leone, "The radiation of a rectangular power-bus structure at multiple cavity-mode resonances," IEEE Trans. Electromagn. Compat., vol. 45 , no. 3, pp. 486-492, Aug. 2003.

[11] J. S. Pak, J. Kim, H. Lee, J. Byun, and J. Kim, "Coupling of throughhole signal via to power/ground resonance and excitation of edge radiation in multilayer PCB," in Proc. IEEE Int. Symp. Electromagn. Compat., Boston, MA, Aug. 2003, pp. 231-235.

[12] C. Wang, J. L. Drewniak, and J. Nadolny, "Anticipating EMI using transfer functions and signal integrity information," in Proc. IEEE Int. Symp. Electromagn. Compat., Boston, MA, Aug. 2003, pp. 695-698.

[13] S. Haga, K. Nakano, and O. Hashimoto, "Reduction in radiated emission by symmetrical power-ground layer stack-up PCB with no open edge," in Proc. IEEE Int. Symp. Electromagn. Compat., Minneapolis, MN, Aug. 2002, pp. 253-257.

[14] T. Harada, H. Sasaki, and T. Kuriyama, "Radiated emission from multilayer PCB with traces placed between power/ground planes," in Proc. IEEE Int. Symp. Electromagn. Compat., Minneapolis, MN, Aug. 2002, pp. 262-267.

[15] I. Novak, "Reducing simultaneous switching noise and EMI on ground/power planes by dissipative edge termination," IEEE Trans. Adv. Packag., vol. 22, no. 3, pp. 274-283, Aug. 1999.

[16] X. Yee, D. M. Hockanson, M. Li, Y. Ren, W. Cui, J. L. Drewniak, and R. E. DuBroff, "EMI mitigation with multilayer power-bus stacks and via stitching of reference planes," IEEE Trans. Electromagn. Compat., vol. 43, no. 4, pp. 538-548, Nov. 2001

[17] S. M. Rao, Time Domain Electromagnetics. New York: Academic, 1999.

[18] G. A. Hjellen, "Including dielectric loss in printed circuit models for improved EMI/EMC predictions," IEEE Trans. Electromagn. Compat., vol. 39, no. 3, pp. 236-246, Aug. 1997.

[19] D. K. Cheng, Field and Wave Electromagnetics, 2nd ed. Reading, MA: Addison-Wesley, 1989.

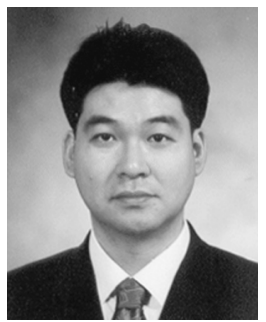

Jun So Pak (S'01-M'05) received the B.S. degree in electrical communication engineering from Hanyang University, Seoul, Korea, in 1998, and M.S. and Ph.D. degrees in electrical engineering from the Korea Advanced Institute of Science and Technology (KAIST), Daejeon, in 2000 and 2005, respectively. His doctoral thesis was a study on the SPICE circuit-type modeling technique (balanced TLM and via coupling model) for the analysis of the power/ground plane noise and edge radiation in high-performance multilayer PCBs and packages.

Since 2005, he has been a JSPS Research Fellow funded by the Japan Society for the Promotion of Science (JSPS) with the High-Density Interconnection Group, National Institute of Advanced Industrial Science and Technology (AIST), Tsukuba, Japan, where he is involved in the development of interconnection techniques for 3-D multichip packaging. His main part of the development is the electrical analysis of the interconnections based on modeling.

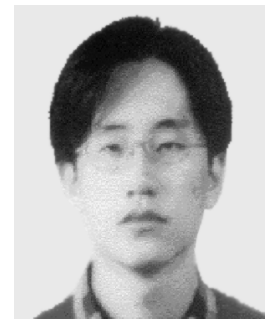

Hyungsoo Kim (S'06) received the B.S., M.S., and $\mathrm{Ph} . \mathrm{D}$. degrees in electrical engineering from the Korea Advanced Institute of Science and Technology (KAIST), Daejon, in 1997, 1999, and 2005, respectively.

In 2005, he joined Hynix Semiconductor Inc., Gyunggi-do, Korea, where he is now Member of Technical Staff with the Advanced Design Team. He has been working with high-speed VLSI interconnections, high-speed digital ICs with minimized EMI radiation, adaptive output driver design, embedded capacitor characterization, and design of power distribution networks on chip, package, and PCB. His current research interest is in DRAM's high-speed interface design considering signal and power integrity.

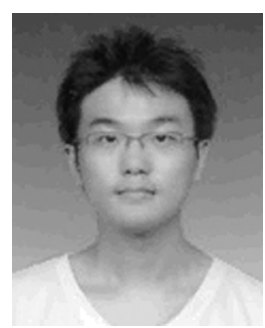

Junwoo Lee (S'00-M'06) received the B.S., M.S., and $\mathrm{Ph} . \mathrm{D}$. degrees in electrical engineering from the Korea Advanced Institute of Science and Technology (KAIST), Daejeon, in 1999, 2001, and 2005, respectively.

In 2002, he was with the Institute of Microelectronics, Singapore, for a one-year internship. In 2005, he joined Hynix Semiconductor Inc., Gyunggi-do, Korea, where he is currently responsible for the design of on-chip power distribution networks. His research interests are in the design of power distribution networks and driver/receiver circuits of high-speed memory.

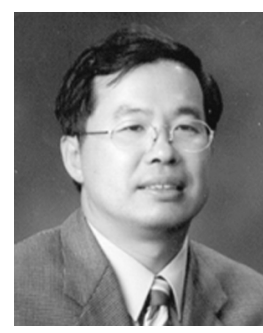

Joungho Kim (M'06) received the B.S. and M.S. degrees in electrical engineering from Seoul National University, Seoul, Korea, in 1984 and 1986, respectively, and the Ph.D. degree in electrical engineering from the University of Michigan, Ann Arbor, in 1993. During his graduate study, he was involved in femtosecond time-domain optical measurement techniques for high-speed device and circuit testing.

In 1993, he joined Picometrix, Inc, Ann Arbor, MI, to work as a Research Engineer, where he was responsible for development of picosecond sampling systems and 70-GHz photoreceivers. In 1994, he joined the Memory Division of Samsung Electronics, Kiheung, Korea, where he was engaged in gigabit-scale DRAM design. In 1996, he moved to the Korea Advanced Institute of Science and Technology (KAIST), Daejeon. He is currently an Associate Professor in the Electrical Engineering and Computer Science Department. Since joining KAIST, his research has centered on modeling, design, and measurement of high-speed interconnection, packages, and PCBs. His research topics especially include design issues of signal integrity, power/ground noise, and radiated emission in high-speed SerDes channel, system-on-package (SoP), and multilayer PCBs. He was on sabbatical leave during academic year from 2001 to 2002 at Silicon Image, Inc., Sunnyvale, CA, as a Staff Engineer. He was responsible for low-noise package design of SATA, FC, and Panel Link SerDes devices. He is the author or coauthor of more than 160 publications in refereed journals and conferences.

Dr. Kim has the Chair or the Co-Chair of the EDAPS workshop since 2002. Currently, he is an Associate Editor of the IEEE TRANSACTIONS ON ELECTROMAGNETIC COMPATIBILITY. 